\title{
Understanding the Loading Dependence of Self-Diffusion in Carbon Nanotubes
}

\author{
S. Jakobtorweihen, ${ }^{1}$ M. G. Verbeek, ${ }^{2}$ C. P. Lowe, ${ }^{2}$ F. J. Keil, ${ }^{1}$ and B. Smit ${ }^{2,3}$ \\ ${ }^{1}$ Chemical Reaction Engineering, Hamburg University of Technology, Eissendorfer Strasse 38, D-21073 Hamburg, Germany \\ ${ }^{2}$ Van 't Hoff Institute for Molecular Sciences (HIMS), University of Amsterdam, Nieuwe Achtergracht 166, \\ 1018 WV Amsterdam, The Netherlands \\ ${ }^{3}$ CECAM (Centre Européen de Calcul Atomique Moléculaire), Ecole Normale Supérieure, \\ 46 Allée d'Italie, 69364 Lyon Cedex 7, France
}

(Received 22 February 2005; published 18 July 2005)

\begin{abstract}
The influence of flexible walls on the self-diffusion of $\mathrm{CH}_{4}$ in an isolated single walled carbon nanotube, as an example, is studied by molecular dynamics simulations. By simulating the carbon nanotube as a flexible framework we demonstrate that the flexibility has a crucial influence on selfdiffusion at low loadings. We show how this influence can be incorporated in a simulation of a rigid nanotube by using a Lowe-Andersen thermostat which works on interface-fluid collisions. The reproduction of the results of a flexible carbon nanotube by a rigid nanotube simulation is excellent.
\end{abstract}

Carbon nanotubes (CNTs) can be aligned in a polymer film to form a well-ordered nanoporous membrane structure [1] which can be incorporated in a macroscopic structure [2] for separation devices. It is therefore of practical interest to understand the diffusive behavior of molecules adsorbed in these materials [3-7]. A particularly interesting observation is the remarkable increase of the diffusion coefficient of simple molecules at low densities observed by Skoulidas et al. [3]. These molecular simulations predict a diffusion coefficient higher than the corresponding gas phase value, resulting in fluxes that are orders of magnitude greater than in crystalline zeolitic membranes [3]. These results have subsequently been reproduced by other groups [8,9] and are explained in terms of the smoothness of the nanotube [3].

From a computational point of view, simulations at the low density limit are surprisingly expensive; one needs an increasingly long nanotube to reach the low density limit. This poses no difficulty if one assumes a rigid substrate. However, if one has a material in which the flexibility cannot be ignored and a full atom simulation of the material is required, the calculation becomes many orders of magnitude more expensive and is completely dominated by the substrate. Therefore, most simulation studies use a rigid lattice.

A novel algorithm that takes the most important aspects of flexibility into account at a fraction of the costs of a fully flexible CNT simulation is presented, resulting in effectively the same diffusivities and other effects as obtained from the flexible CNT simulations. This algorithm can be applied to other confined systems (zeolites, ion channels, membranes, etc.).

Interestingly, whether or not it is reasonable to assume a rigid lattice in adsorption [10] or diffusion studies [11] is far from being understood. An obvious hypothesis would be that only in the case of narrow passages is flexibility very important, while in the case of gas molecules in carbon nanotubes, or other nanoporous materials, a rigid lattice is a very reasonable assumption. As we will show in this Letter, this assumption is the explanation of the remarkable increase of the diffusion coefficient at low loading. Molecular dynamics simulations of a fully flexible nanotube give a diffusion coefficient that is more than 1 order of magnitude lower than that reported by Skoulidas et al. [3].

It is instructive to consider a collision of a gas molecule with a wall in detail. We can distinguish two extremes; a perfectly smooth wall or a very corrugated wall. If the wall is perfectly smooth, the collisions of the gas particles are completely elastic and the component of the velocity parallel to the wall remains constant and hence the parallel motion would be ballistic. Diffusive behavior can then only arise from collisions with other gas particles, which is negligible at low density. If, on the other hand, we have a sufficiently corrugated wall, these corrugations introduce sufficient randomness in the collisions to observe diffusive behavior without the need for collisions with other adsorbed gas particles. Diffusion in zeolites is a typical example of the latter. CNTs are very smooth and are therefore closer to a perfectly smooth wall. However, in a real nanotube there are always some thermal fluctuations of the wall atoms that interact with the adsorbed molecules. These fluctuations are a cause of randomness that will lead to diffusive behavior which should dominate the ballistic motion at sufficiently low densities; i.e., one would observe a constant diffusion coefficient in the limit of low density. This argument could suggest that the low density results of Skoulidas et al. [3] are not physical. However, as this argument is only qualitative, this plateau could occur (far) below the density that is studied by Skoulidas et al. [3]. To test this hypothesis we performed molecular dynamics simulations of methane in a flexible isolated single walled $(20,0)$ CNT.

The fully flexible CNT is modeled with the potential given in [12]. Methane is modeled as a united-atom with the Lennard-Jones (LJ) parameters taken from [13] and the 
carbon LJ parameters taken from Ref. [14]. Each simulation was performed with 64 methane molecules. To simulate different loadings, the carbon nanotube length is adjusted. To confirm that 64 fluid particles are sufficient, some simulations have been repeated with twice as many molecules and with double the tube length. These simulations showed that the choice of 64 guest molecules is justified. For simulations containing a rigid CNT, a time step $\Delta t$ of 2 fs was used. For the flexible CNT, the reversible reference system propagation algorithm was used, with the short time step $\left(\Delta t_{S}=0.5 \mathrm{fs}\right)$ for bond stretching potential and the long time step $\left(\Delta t_{L}=2 \mathrm{fs}\right)$ for the remainder of the interactions. Only the atoms of the flexible CNTs are coupled to a Nosé-Hoover thermostat $[15,16]$. The methane molecules are thermostated indirectly via collision with the atoms of the CNT. All self-diffusion coefficients $D_{S}$ are calculated for $300 \mathrm{~K}$ and only for motion parallel to the pore axis (see Fig. 1) by means of the Einstein equation [16]. Errors are obtained by averaging over five independent simulations. In the figures, the error bars are not shown when they are smaller than the symbol size.

Self-diffusion coefficients were calculated for loadings ranging from near saturation down to nearly zero loading. Simulations were performed using the methodology of Skoulidas et al., that is, with a rigid tube and a Nosé-Hoover thermostat coupled to the $\mathrm{CH}_{4}$ molecules. Although we used a slightly different model, Fig. 2 shows that our results for the rigid CNT are in excellent agreement with the results of Skoulidas et al.. These simulations confirm the pronounced ( 3 orders of magnitude) increase of the diffusion coefficient with decreasing pressure.

We observe a completely different loading dependence for a fully flexible CNT. At high loading intermolecular collisions determine the diffusion coefficient and one would expect little influence, which is indeed the case, as is shown in Fig. 2. When the pressure decreases, an increase in diffusion is observed, but this increase is far less pronounced than for the rigid CNT. By a further reduction of the density, the diffusion coefficient becomes constant again. This plateau region is not observed when simulating a rigid CNT, neither in this work, nor in other publications $[3,8,9,17]$. By lowering the loading, the number of fluidfluid collisions approaches zero. The diffusion becomes independent of the loading and only CNT-fluid collisions have an influence on the diffusion. This independence is
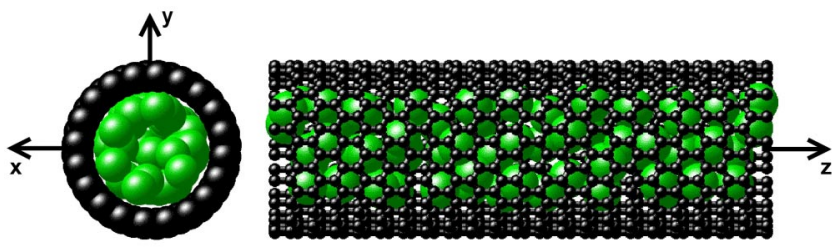

FIG. 1 (color online). Sketch of the carbon nanotube methane system. much more realistic than the results obtained by using a rigid CNT. A comparison with the adsorption isotherm shows that the results start to deviate at the transition to the Henry regime (not shown here). By definition, adsorbate-adsorbate interactions do not take place in the Henry regime [18].

Clearly, our simulations of the flexible nanotube show that the assumption of a rigid lattice leads to unphysical results. The most important contribution that is ignored in the rigid structure is that at each collision of a gas molecule with the wall there is an exchange of energy and momentum. Because of the thermal motion of the carbon atoms in the tube, some randomness is induced in the behavior of the gas molecules that is not taken into account in the rigid tube simulations. Because of the large number of carbon atoms, these fully flexible CNT simulations are very expensive. It is therefore important to develop an algorithm in which the CPU time is reduced.

The central idea of our novel algorithm is that, due to the collisions with the wall, some randomness is introduced into the system. At the level of the flexible framework simulations, this randomness, related to thermal fluctuations, is treated exactly, but this effect can also be mimicked stochastically, such that each gas molecule that hits an atom of the wall has a probability of exchanging momentum. This is similar in spirit to the Lowe-Andersen (LA) thermostat for constant temperature (bulk) dissipative particle dynamics simulations $[19,20]$. The LA thermostat is modified such that it works on interface-fluid collisions. The parameters of this Lowe-Andersen interface-fluid collision (LA-IFC) thermostat can be obtained from simulations of a flexible CNT. In this way, the influence of a flexible interface is introduced in a simulation of a rigid CNT, which is considerably faster than simulating a flexible CNT.

The LA thermostat works on pairs of particles. A new relative velocity for each pair of particles is taken from a

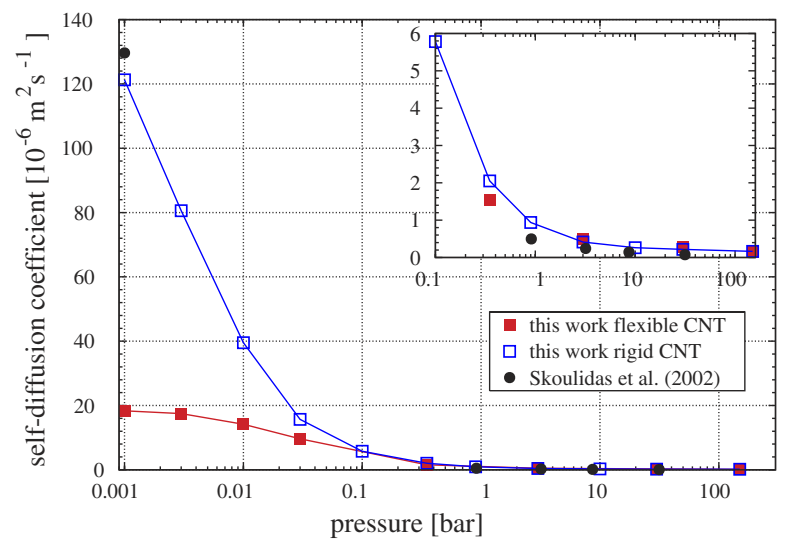

FIG. 2 (color online). Self-diffusion coefficients of $\mathrm{CH}_{4}$ in a $(20,0)$ carbon nanotube calculated with a rigid CNT and with a flexible CNT. The inset is a section of the data on an expanded scale. Lines are added to guide the eye. 
Maxwell distribution, so that the total momentum is conserved [19]. In real systems, heat is transferred via the system boundaries only, i.e., for the present system via a collision of a gas molecule with the CNT. This can be mimicked stochastically via a LA thermostat that only acts when there are fluid-wall collisions. As in the simulations the carbon atoms are kept fixed, only the velocity of a carbon atom is changed. If the shortest distance $d$ between a particle and the wall (defined by the position of the carbon nuclei) is shorter than $r_{\mathrm{LA}}$, the velocity of a molecule is updated with a probability $\Gamma \Delta t$ and the new velocity is given by

$$
v_{f, \alpha}^{\text {new }}=v_{f, \alpha}^{\text {old }}+\frac{\mu}{m_{f}}\left(\xi_{1} \sqrt{\frac{k_{B} T}{m_{C}}}-v_{f, \alpha}^{\text {old }}\right)+\xi_{2} \sqrt{\frac{\mu k_{B} T}{m_{f}^{2}}}
$$

where $\alpha$ represents the direction of the velocity $v, m$ is the mass of a particle, the subscript $f$ indicates a fluid molecule, whereas the subscript $C$ stands for carbon, $\mu$ is the reduced mass, $k_{B}$ is the Boltzmann constant, $T$ is the temperature, and $\xi_{1}$ and $\xi_{2}$ are independent random numbers from a Gaussian distribution with zero mean and unit variance.

The collision frequency $\Gamma$ and the LA radius $r_{\mathrm{LA}}$ have to be chosen such that our algorithm optimally describes a truly flexible CNT. The CNT geometry (see Fig. 1) results in a different mobility of the carbon atoms in different directions [21] and hence the collision frequency should be different for collisions orthogonal and parallel to the tube axis. The $x$ and $y$ (orthogonal) components of the particle velocity are updated [see Eq. (1)] with a probability $\Gamma_{x y} \Delta t$, and the $z$ (parallel) component is updated with a probability $\Gamma_{z} \Delta t$.

The parallel collision frequency can be obtained from the velocity autocorrelation function (VACF). Within the Markovian approximation, neglecting correlation effects that are normally small in a gas [22], the Langevin equation relates the decay of the VACF to a friction coefficient $\gamma$ :

$$
\left\langle v_{z}(0) v_{z}(t)\right\rangle=\left(k_{B} T / m_{f}\right) \exp \left[-\gamma t / m_{f}\right] .
$$

This friction coefficient is directly related to our collision rule (1): $\gamma=\Gamma \mu$. Because in our simulation collisions only take place within the LA radius, we have: $\Gamma_{z}\left(r_{\mathrm{LA}}\right)=$ $\Gamma_{z}\left(r_{\mathrm{LA}}=r_{\mathrm{CNT}}\right) / P\left(r_{\mathrm{LA}}\right)$, where $P$ gives the probability that a molecule is located in a spherical shell specified by $r_{\mathrm{LA}}$. $P$ can be calculated from the radial distribution function, which in turn can be calculated in a molecular dynamics or Monte Carlo simulation.

We use these expressions to obtain the parallel collision frequency. The VACF requires a simulation with a fully flexible CNT. Because we only need the short time limit at vanishing density, to exclude gas-gas collision, such a completely flexible simulation is relatively cheap and has to be done only once for a given temperature. The parallel collision frequency $\Gamma_{z}$ is fitted to a VACF in the range 0 $100 \mathrm{ps}$, and the particle dynamics are followed for $8 \mathrm{~ns}$. A comparison with the results of a much longer simulation justifies these choices.

As the VACF immediately drops to zero in the orthogonal direction, a different procedure is used to obtain the orthogonal collision frequency $\Gamma_{x y}$. This frequency is obtained by fitting to a heating experiment, in which we change the temperature of the flexible CNT at $t=0$ and monitor the time it takes the gas to reequilibrate at the new temperature. As we only thermostat the atoms of the CNT, the heating of the adsorbed gas takes place via collisions with the atoms of the flexible CNT. As these heating curves are different for the orthogonal and parallel directions, they also provide a check for the consistency of the parameters that have been obtained from the fit to the VACF. Figure 3 shows that the LA thermostat nicely reproduces the heating curves of the flexible CNT, also for the parallel direction for which the value of $\Gamma_{z}$ is obtained from the VACF.

For the approach introduced, it is necessary to predefine a Lowe-Andersen radius $r_{\mathrm{LA}}$. As this choice is to some extent arbitrary it is important to ensure that any reasonable description should give consistent results. To investigate the influence of the parameters, the loading dependence of self-diffusion was calculated with four different parameter sets (see legend of Fig. 4). As expected, the frequencies $\Gamma$ increase with decreasing LA radius, because less particles take part in the thermalization process.

By definition the LA-IFC thermostat should only work on fluid-interface collisions and a reasonable choice of $r_{\mathrm{LA}}$ is one where only particles near the wall are thermalized. The LA radius of $4 \AA$ represents the minimum of the $\mathrm{LJ}$ carbon- $\mathrm{CH}_{4}$ interaction energy and the LA radius of $3.6 \AA$

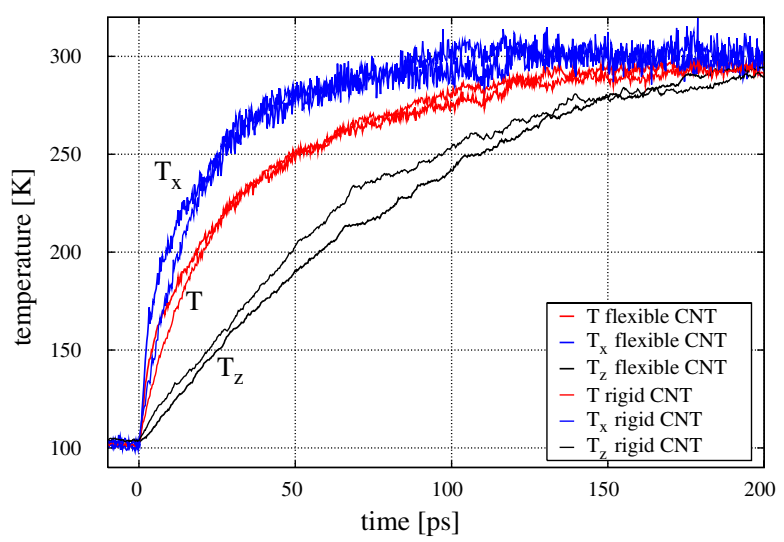

FIG. 3 (color online). Heating curves for heating ideal $\mathrm{CH}_{4}$ in a $(20,0)$ carbon nanotube from 100 to $300 \mathrm{~K}$, calculated with a flexible CNT (thick lines) or with a rigid CNT (thin lines) by using the LA thermostat (as described in the text). The LA curves are calculated with the $r_{\mathrm{LA}}=7.8 \AA$ data set (see legend of Fig. 4). $T$ is the conventional temperature $T=f\left(v_{x}, v_{y}, v_{z}\right)$, $T_{x}$ the temperature for the $x$ direction and $T_{z}$ for the $z$ direction. The temperature switch is defined to take place at a time $t=$ 0 ps. All heating curves are averages over 100 independent simulations. 


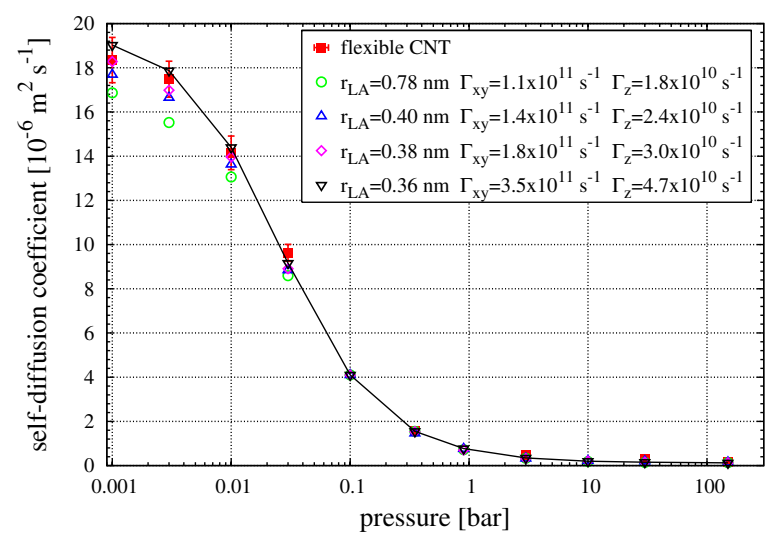

FIG. 4 (color online). Self-diffusion coefficients obtained with the different LA parameter sets (rigid CNT), compared to results obtained with a flexible CNT. The line is added to guide the eye.

represents the maximum of the radial distribution function. Within these parameter sets we can nicely reproduce the flexible simulations (see Fig. 4), which illustrates that our method gives very reasonable results that do not crucially depend on the parameters that have to be specified. At this point it is important to note that if we take such a large value of the radius $\left(r_{\mathrm{LA}}=7.8 \AA\right.$ ) that all particles take part in the thermalization no matter where they are located, we do see systematic deviations. But even in this extreme case the deviation is less than $10 \%$. Therefore, our local LoweAndersen thermostat correctly incorporates the influence of the flexibility of the CNT on the diffusion and also the heat transfer is accurately reproduced. Once the parameters of the LA-IFC thermostat have been determined the algorithm is equally efficient as a rigid CNT simulation.

In the present work we have shown that the flexibility of a smooth pore cannot be neglected in the low loading regime. Simulations, in CNTs or certain other nanoporous materials, that neglect this influence $[3,8,9,17]$ can lead to an overprediction of the self-diffusion coefficient by orders of magnitude. The computed self-diffusion coefficients in a flexible CNT are still higher than for zeolites, but not 2 orders of magnitude as suggested by a simulation in a rigid CNT. The diffusion coefficients are also significantly lower than the corresponding diffusion coefficients in the gas phase.

A novel very efficient algorithm was introduced which incorporates stochastically the effects of a flexible CNT into a simulation using a rigid CNT, such that computations employing a flexible CNT can be avoided. These simulations are significantly faster (factor 25 to 5 orders of magnitude; these estimates depend on loading and details of the implementation) and lead to the same self-diffusion coefficients and give the same heat transfer across the rigid interface as across a flexible interface.

This work was supported by the Deutsche Forschungsgemeinschaft (DFG) in priority program SPP 1155 . We are grateful to David Dubbeldam and Edith Beerdsen for fruitful discussions.

*Corresponding author: jakobtorweihen@tuhh.de

[1] B. J. Hinds, N. Chopra, T. Rantell, R. Andrews, V. Gavalas, and L. G. Bachas, Science 303, 62 (2004).

[2] A. Srivastava, O. N. Srivastava, S. Talapatra, R. Vajtai, and P. Ajayan, Nat. Mater. 3, 610 (2004).

[3] A. I. Skoulidas, D. M. Ackerman, J. K. Johnson, and D. S. Sholl, Phys. Rev. Lett. 89, 185901 (2002).

[4] C. Wei and D. Srivastava, Phys. Rev. Lett. 91, 235901 (2003).

[5] O. G. Jepps, S. K. Bhatia, and D. J. Searles, Phys. Rev. Lett. 91, 126102 (2003).

[6] G. Arya, H. C. Chang, and E. J. Maginn, Phys. Rev. Lett. 91, 026102 (2003).

[7] V.P. Sokhan, D. Nicholson, and N. Quirke, J. Chem. Phys. 120, 3855 (2004).

[8] D. Cao and J. Wu, Langmuir 20, 3759 (2004).

[9] G. Arora, N. J. Wagner, and S. I. Sandler, Langmuir 20, 6268 (2004).

[10] T. J. H. Vlugt and M. Schenk, J. Phys. Chem. B 106, 12757 (2002).

[11] P. Demontis and G. B. Suffritti, Chem. Rev. 97, 2845 (1997).

[12] J.H. Walther, R. Jaffe, T. Halicioglu, and P. Koumoutsakos, J. Phys. Chem. B 105, 9980 (2001).

[13] M. G. Martin and J. I. Siepmann, J. Phys. Chem. B 102, 2569 (1998).

[14] W. A. Steele, The Interaction of Gaes with Solid Surfaces (Pergamon, Oxford, 1974).

[15] G. J. Martyna, M.E. Tuckerman, D. J. Tobias, and M. L. Klein, Mol. Phys. 87, 1117 (1996).

[16] D. Frenkel and B. Smit, Understanding Molecular Simulations, From Algorithms to Applications (Academic Press, San Diego, 2002).

[17] D. M. Ackerman, A. I. Skoulidas, D. S. Sholl, and J. K. Johnson, Mol. Simul. 29, 677 (2003).

[18] F. Rouquerol, J. Rouquerol, and K. Sing, Adsorption by Powders \& Porous Solids (Academic Press, San Diego, 1999).

[19] C.P. Lowe, Europhys. Lett. 47, 145 (1999).

[20] C.P. Lowe and M.W. Dreischor, in Novel Methods in Soft Matter Simulations, edited by M. Karttunen, I. Vattulainen, and A. Lukkarinen Lecture Notes in Physics, Vol. 640 (Springer, Berlin, 2004), pp. 39-68.

[21] V. P. Sokhan, D. Nicholson, and N. Quirke, J. Chem. Phys. 117, 8531 (2002).

[22] R. Zwanzig, Nonequilibrium Statistical Mechanics (Oxford University Press, Oxford, 2001). 